\title{
PRIMER HALLAZGO DE MYXINE CIRCIFRONS GARMAN 1899 (AGNATHA: MYXINIDAE) EN LA COSTA DEL PACÍFICO DE COLOMBIA
}

\section{FIRST RECORD OF MYXINE CIRCIFRONS GARMAN 1899 (AGNATHA: MYXINIDAE) FROM THE PACIFIC COLOMBIAN COAST}

\author{
Efraín A. Rubio ${ }^{2}$, Milton J. Pedraza ${ }^{1}$ \& Luis A. Zapata ${ }^{3}$ \\ ${ }^{1}$ Departamento de Biología, Magíster en Ciencias mención Pesquerías, Universidad de Concepción (Chile) \\ Email: mipedraz@udec.cl. \\ ${ }^{2}$ Departamento de Biología, Facultad de Ciencias, Universidad del Valle (Colombia). \\ ${ }^{3}$ Instituto Nacional de Pesca y Acuicultura (INPA). Apartado aéreo 10742, Buenaventura (Colombia)
}

\begin{abstract}
RESUMEN
Los peces bruja Myxine circifrons Garman 1899 habitan sobre fondos fangosos en la zona preabisal, capturándose principalmente entre los 500 y 1.500 m de profundidad; su distribución geográfica está ligada a las regiones sub-tropicales prefiriendo aguas relativamente frías. Este trabajo registra por primera vez en aguas del océano Pacífico colombiano a $700 \mathrm{~m}$ de profundidad la presencia de los agnathos vivientes más primitivos en los océanos del mundo.
\end{abstract}

Palabras claves: Primer registro, Myxine circifrons, Pacífico colombiano.

\section{ABSTRACT}

This hagfish species in habiting muddy bottom, between 500 and $1500 \mathrm{~m}$ depth; shows a worldwide distribution occupying all sub-tropical oceans, with preference in cold waters. This paper reports the first catch record of Myxine circifrons Garman 1899, from the Colombian Pacific Ocean.

Keywords: First record, Myxine circifrons, Colombian Pacific Ocean.

\section{INTRODUCCION}

Los hagfish fueron alguna vez organismos extremadamente diversos, con una amplia distribución geográfica. En la actualidad, aunque su ancestro no sobrevive, es importante reconocer su éxito como grupo, ya que están presentes en el océano desde hace unos 140 millones de años. Los hagfish están representados por seis géneros y más de 32 especies, todas contenidas dentro de una sola familia: Myxinidae, todas restringidas al hábitat marino con una distribución cosmopolita incluyendo los golfos de México y Panamá (Nelson 1994).

Las especies de hagfish están representadas en los géneros Myxine, Neomyxine, Paramyxine, Eptatretus,
Notomyxine (1 sp.) y Nemamiyxine (2 spp.); estos dos últimos géneros, junto con el género Myxine, conforman la subfamilia Myxininae, mientras que los géneros restantes (Paramyxine, Epatatretus) conforman la subfamilia Eptatretinae (Nelson 1994). El género Myxine incluye 19 spp., las cuales están caracterizadas porque las branquias son aferentes a un saco dúctil que descarga a una sola apertura branquial a cada lado del cuerpo y por la ausencia de estrías en la parte cefálica (Wisner \& McMillan 1995). Los representantes de la familia Myxinidae son reconocidos como criaturas únicas, catalogados como peces carroñeros que pueden, además, alimentarse de peces e invertebrados marinos vivos. Al ser carroñeros cumplirían un importante papel en la limpieza del 
ambiente en el cual viven (Jansen 1966; Kato 1990). La captura de dos ejemplares de Myxine circifrons en el área sur del Pacífico de Colombia constituye un importante hallazgo para la zona entre el Golfo de Panamá e Islas Galápagos y es, a la vez, una contribución importante al conocimiento de las especies marinas costeras de aguas profundas.

\section{MATERIALES Y METODOS}

La recolecta de los ejemplares se realizó a bordo de la embarcación coreana "Sam Hae 101" cuya pesquería objetivo es el camarón cabezón de profundidad Heterocarpus hostilis Faxon 1893, utilizando como arte trampas metálicas recubiertas con malla, armadas con carduma, Cetengraulis mysticetus Günther 1866 como carnada; las cuales llegan a profundidades entre 600 y $900 \mathrm{~m}$ con una duración promedio en el agua de 13 horas.

Después de la captura los ejemplares fueron fijados en formalina al $10 \%$, para su traslado al laboratorio de Ictiología de la sección de Biología Marina en la Universidad del Valle.

La identificación se realizó utilizando las descripciones y claves de Garman (1899), Chirichigno (1974, 1978); Nelson (1994) y Fernholm (1998). Los ejemplares se conservaron en alcohol y depositaron en la colección de Ictiología de referencia de la sección de Biología Marina en la Universidad del Valle (CIRUV) en la ciudad de Cali y con número de catálogo 95002.

Los métodos empleados para el conteo y medición en los ejemplares es el presentado por Fernholm (1998). Todas las medidas son en mm, y las proporciones del cuerpo son presentadas con base a longitud total (Tabla I).

\section{RESULTADOS}

Nombres vernaculares: Castellano: pez bruja; lamprea babosa. Inglés: hagfish; whiteface hagfish.

Material examinado: Dos ejemplares () de 383 y $400 \mathrm{~mm}$ de longitud total (Figura 1), recolectados el 12 de julio de 1995 . Lance $\mathrm{N}^{\circ} 15$ posición geográfica

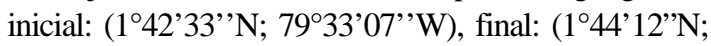
79²9'03”'W), $700 \mathrm{~m}$ de profundidad; recolector: Biólogo M. Pedraza.

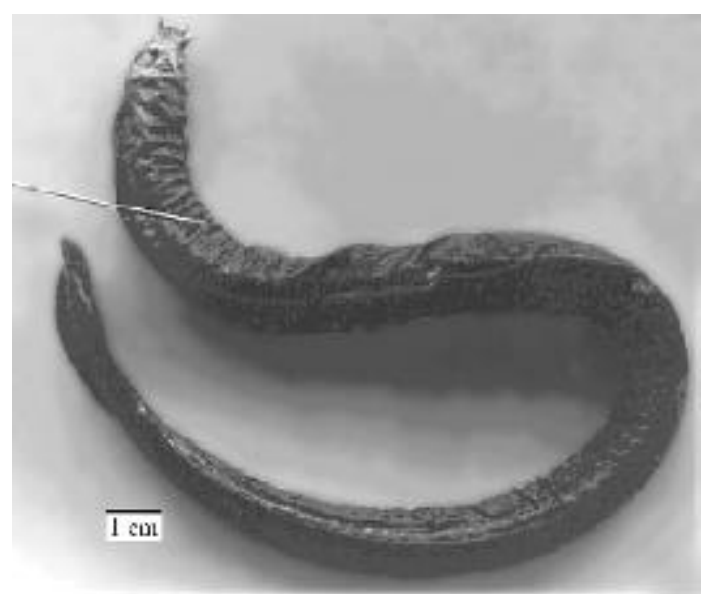

FIGURA 1. Individuo adulto de Myxine circifrons (400mm Lt).

FIGURe 1. Adult of Myxine circifrons (400mm Lt)

CARACTERES Distintivos: Los conteos y las medidas proporcionales se presentan en la Tabla I. Los ejemplares analizados se caracterizan por poseer un cuerpo robusto, no alargado; rostro corto y ligeramente redondeado; 13 odontoides linguales en la primera serie o serie anterior, de los cuales los 3 primeros están fusionados en la base; el paquete de branquias son eferentes a una sola abertura branquial ubicada a cada lado de su cuerpo, justo antes de la inserción del pliegue-aleta ventral; 84 y 86 poros totales respectivamente, 23 y 24 poros prebranquiales, 51 y 52 poros entre la abertura branquial y la cloaca, 10 poros posteriores a la cloaca; cabeza pequeña; boca sin mandíbulas; 4 barbos cercanos a la boca; cuerpo de color negro uniforme; cabeza y tentáculos pálidos; los individuos capturados presentan semejanzas y diferencias en los conteos y medidas proporcionales con los datos de Garman (1899), Chirichigno (1974) y Wisner \& McMillan (1995), lo cual podría indicar variaciones intraespecíficas. Esto se puede observar en la Tabla I.

HÁBITAT: El pez bruja es una especie bentodemersal profunda muy poco conocida, siendo nuestros ejemplares fauna acompañante en las capturas del camarón de profundidad (H. hostilis); recolectados entre $700 \mathrm{y}$ $1.860 \mathrm{~m}$ de profundidad, sobre fondos fangosos a temperaturas de $3-4^{\circ} \mathrm{C}$.

Pesca y utilización: La mayoría de las especies no revisten ningún peligro, la pesquería de estos peces (no en Colombia) se centra en el aprovechamiento 
Gayana 69(1), 2005

TABla I. Análisis morfométrico y méristico de los individuos de Myxine circifrons reportados para la costa pacífica colombiana y data registrada por otros autores en varias localidades del Pacífico Oriental para ejemplares de la misma especie. Garman (1899), Chirichingo (1978) y Wisner \& McMillan (1995).

TABLE I. Morfometric and measurements analysis of individuals Myxine circifrons report Pacific coast colombiana and other report data in several localities the Pacific Easter. Garman (1899), Chirichingo (1978) y Wisner \& McMillan (1995).

\begin{tabular}{|c|c|c|c|c|c|c|}
\hline Localidad & $\begin{array}{l}\text { Colombia } \\
1^{\circ} 42^{\prime} \mathrm{N} ; \\
79^{\circ} 33^{\prime} \mathrm{W}\end{array}$ & $\begin{array}{c}\text { Colombia } \\
1^{\circ} 42^{\prime} \mathrm{N} ; \\
79^{\circ} 33^{\prime} \mathrm{W}\end{array}$ & $\begin{array}{c}\text { Panamá } \\
\text { 7³0’N; } \\
\text { 78³9’W } \\
\text { Garma } 1899\end{array}$ & $\begin{array}{c}\text { Perú } \\
3^{\circ} 47^{\prime} \mathrm{S} ; \\
81^{\circ} 28^{\prime} \mathrm{W} \\
\text { Chirichigno } \\
1978 \\
\end{array}$ & $\begin{array}{c}\text { Perú } \\
1^{\circ} 08^{`} \mathrm{~S} ; \\
72^{\circ} 11^{\prime} \mathrm{W} \\
\text { Chirichigno } \\
1978\end{array}$ & $\begin{array}{c}\text { Easterm } \\
\text { Pacific } \\
\text { Wisner \& } \\
\text { McMillan } \\
1995 \\
\end{array}$ \\
\hline Numero de ejemplares & 1 & 1 & 1 & 1 & 1 & 572 \\
\hline Longitud total (mm) & 383 & 400 & 467 & 543 & 355 & $125-650$ \\
\hline $\begin{array}{l}\text { Long Pre-branquial }(\mathrm{mm}) \\
(\% \text { en } \mathrm{Lt})\end{array}$ & $\begin{array}{c}113.7 \\
(29,7 \%)\end{array}$ & $\begin{array}{c}114.8 \\
(28,7 \%)\end{array}$ & $\begin{array}{c}145.7 \\
(31,2 \%)\end{array}$ & $\begin{array}{c}154.7 \\
(28,5 \%)\end{array}$ & $\begin{array}{c}107.9 \\
(30,4 \%)\end{array}$ & $290-300$ \\
\hline Pre-branquial en Lt(veces) & 3,36 & 3,48 & 3,2 & 3,5 & 3,28 & --- \\
\hline Altura cuerpo (mm) & 19,9 & 18,4 & 25,7 & 26,1 & 21 & --- \\
\hline$(\%$ en $\mathrm{Lt})$ & $(5,1 \%)$ & $(4,6 \%)$ & $(5,5 \%)$ & $(4,8 \%)$ & $(5,9 \%)$ & \\
\hline Altura cuerpo en $\mathrm{L}_{\mathrm{t}(\text { veces })}$ & 19,2 & 21,7 & 18,2 & 20,8 & 16,9 & --- \\
\hline Altura pliegue dorsal (mm) & 8,0 & 8,0 & --- & 12,0 & 8,0 & --- \\
\hline$(\%$ en $\mathrm{Lt})$ & $(2,1 \%)$ & $(2,0 \%)$ & & $(2,2 \%)$ & $(2,2 \%)$ & \\
\hline Pliegue dorsal en $\mathrm{L}_{\mathrm{t} \text { (veces) }}$ & 47,8 & 50 & --- & 45,2 & 44,3 & --- \\
\hline Longitud del tronco $(\mathrm{mm})$ & 217 & 219,2 & --- & --- & --- & --- \\
\hline Longitud de caudal (mm) & 52,3 & 66 & --- & --- & --- & --- \\
\hline $\mathrm{N}^{\mathrm{o}}$ poros Pre-branquiales & 23 & 24 & 23 & 26 & 22 & 23 \\
\hline $\mathrm{N}^{\mathrm{o}}$ poros en tronco & 51 & 52 & --- & 57 & 54 & 55 \\
\hline $\mathrm{N}^{\circ}$ poros post ano & 10 & 10 & 11 & 10 & 10 & 10 \\
\hline $\mathrm{N}^{\circ}$ poros totales & 84 & 86 & --- & 93 & 86 & 88 \\
\hline $\mathrm{N}^{\mathrm{o}}$ dientes fusionados anterior & 3 & 3 & --- & 3 & 3 & --- \\
\hline Conteo pliegue dorsal & 89 & 92 & 89 & 95 & 96 & --- \\
\hline Conteo pliegue anal & 40 & 41 & 42 & 40 & 40 & --- \\
\hline
\end{tabular}

de la piel, que es extremadamente fuerte y flexible; comercializada mundialmente como piel de anguila.

DistribuCión GeOgRÁficA: Pacífico Oriental y Central, Islas Galápagos, desde San Francisco, California hasta la zona norte-central de Chile (Wisner \& McMillan 1995); Localidad tipo Golfo de Panamá (07³0’36”N; 78³9’ W) a 1.336 metros de profundidad.
FAUnA ACOMPAÑANTE: La fauna que también fue capturada incidentalmente en las faenas de pesca del camarón de aguas profundas, en la zona que se halló el pez bruja, está compuesta por peces de las familias Nemichthyidae, Uranoscopidae, Scorpaenidae, Squalidae, Congridae, Macrouridae, Ogcocephalidae y Zoarcidae. 


\section{DISCUSION}

Desde la descripción original de Myxine circifrons basada en un ejemplar de $467 \mathrm{~mm}$ del longitud total recolectado en la "estación Albatross 3395" de golfo de Panamá (7³0’36"N; 78³9’W) a 1336 metros de profundidad sobre suelo rocoso (Garman 1899), no se había vuelto a capturar esta especie en aguas del Pacífico Tropical, debido a la preferencia de esta por las aguas templadas y frías. Se han reportado ejemplares sobre la costa peruana, Islas Galápagos y el Golfo de California, comúnmente en la zona preabisal profunda entre los 700 y $1.100 \mathrm{~m}$ (Chirichigno 1974,1978; Chirichigno \& Cornejo 2001; Wisner \& McMillan 1995). Siendo nulos los reportes en las aguas del Pacífico Colombiano, a pesar de que estos organismos se enmarcan en un amplio rango batimétrico y de distribución geográfica (Nelson 1994).

Si bien es cierto que Garman (1899 y con posterioridad otros investigadores informaron del conteo de radios en la aleta dorsal, cabe notar que una de las características de la familia Myxinidae es la carencia de aleta dorsal. Esta confusión es frecuente debido a que el pliegue que se extiende por la región dorsal de estos organismos es en realidad uno prolongación de su aleta caudal. En este trabajo se incluye los recuentos sobre esta prolongación (Tabla I) con el nombre de pliegue dorsal, con el fin de realizar comparaciones de este aspecto a partir de especímenes registrados con anterioridad, incluyendo el tipo utilizado para la descripción de la especie.

Las diferencias observadas en la proporción de medidas corporales en los individuos analizados con respecto a registros hechos por otros autores (Tabla I), no representan diferencias significativas que puedan indicar variaciones a nivel específico o intraespecífico. Esto podría ser resultado de mediciones subjetivas (diferencias en precisión de los elementos de medición) empleadas en las distintas investigaciones de referencia. La presencia de ciclóstomos en aguas sudamericanas constituye un tipo de información muy valiosa, tanto por los aspectos biológicos que se puedan clarificar de las distintas especies, como por las contribuciones a nivel biogeográfico, ecológico, etológico y otra serie de aspectos igualmente relevantes. Este trabajo contribuye en los aspectos biogeográficos de la especie, ya que, además de su registro en aguas colombianas, confirma su presencia dentro de la distribución geográfica sugerida por Chirichigno (1974) y Winser \& McMillan (1995) para estos organismos.

\section{AGRADECIMIENTOS}

Se agradece al Instituto Nacional de Pesca y Acuicultura, INPA, por la obtención de los ejemplares dentro del proyecto "Evaluación del camarón de profundidad (Heterocarpus spp.) en el Pacífico colombiano" y Departamento de Biología de la Universidad del Valle, por la utilización de sus instalaciones.

\section{BIBLIOGRAFIA}

Chirichigno, N. 1974. Clave para identificar los peces marinos del Perú. Vol. $\mathrm{N}^{\circ}$ 44. Instituto del Mar del Perú-IMAPRE. Callao. 387 p.

. 1978. Nuevas adiciones a la ictiofauna marina del Perú. Informe $\mathrm{N}^{\circ}$ 46. Instituto del Mar del PerúIMAPRE. Callao. 109 p.

Chirichigno, N. \& M. R. Cornejo. 2001. Catálogo comentado de los peces marinos del Perú. Instituto del Mar del Perú (IMAPRE). Publicación especial.

Fernholm, B. 1998. Hagfish systematics. In: J.M. Jorgensen, J.P. Lomholt, R.E. Weber \& H. Malte (eds.). The biology of hagfish. Champan \& Hall, London. 578 p.

Garman, S. 1899. Reports of an exploration of west coast of Mexico, Central and South America and the Galapagos Island, in charge of Alexander Agassiz, by the U.S. Fish Commission Steamer "Albatross", during 1891, Lieut. Cmdr. Z. L. Tanner, U.S.N. commanding. XXVI: The fishes. Memorian Museum Comp. Zoological. Harvard University 24: $1-431$.

JANSEn, D. 1996. The hagfish. Sci. Amer., 214: 82-90.

Kato, S. 1990. Report on the biology of Pacific hagfish, Eptatretus stouti, and development of its fishery in California. National Marina fisheries Service, Tiburon, CA, Technical report. 39pp.

Nelson, J.S. 1994. Fishes of the world, $3^{\text {rd }}$ ed. John Wiley and Sons, Inc., New York. 316p.

WisNer, L.R \& McMillan, C.B. 1995. Review of new world hagfish of the genus Myxine (Agnatha, Myxinide) with descriptions of nine new species. Fishery Bulletin 93: 530-550. 